\title{
Beneficios del ejercicio físico en la sesión de hemodiálisis. Una revisión sistemática
}

\author{
Carmen Ma Jiménez-Prieto미, Rosa González-Tamajón' ${ }^{1}$, Rodolfo Crespo-Montero ${ }^{1,2,3}$ \\ ${ }^{1}$ Departamento de Enfermería. Facultad de Medicina y Enfermería. Universidad de Córdoba. Córdoba. España \\ ${ }^{2}$ Servicio de Nefrología. Hospital Universitario Reina Sofía de Córdoba. Córdoba. España \\ ${ }^{3}$ Instituto Maimónides de Investigación Biomédica de Córdoba. Córdoba. España
}

Jiménez-Prieto CM, González-Tamajón R, Crespo-Montero R. Beneficios del ejercicio físico en la sesión de hemodiálisis. Una revisión sistemática. Enferm Nefrol. 2020 Jul-Sep;23(3):233-243

\section{Resumen}

Objetivo: El objetivo principal de esta revisión fue conocer los principales beneficios del ejercicio físico durante la sesión de diálisis, en los pacientes en programa de hemodiálisis periódicas.

Metodología: Se llevó a cabo una revisión sistemática a través de las bases de datos Pubmed, Science Direct y Google Académico. Se incluyeron artículos científicos en inglés y español, y se analizaron los artículos que trataban sobre ejercicio físico en la sesión de hemodiálisis. Se excluyeron aquellos artículos que no presentaran resultados y en los que el ejercicio físico fuese domiciliario.

Resultados: Se incluyeron 18 artículos publicados entre 2015 y 2019. Todos los artículos fueron de diseño experimental. Se encontró una mejoría significativa de la fuerza muscular, al igual que de la capacidad física y de de la sintomatología depresiva. En menor medida, se observó una disminución de la presión arterial, disminución del proceso inflamatorio, mejoría de los valores nutricionales y mejora de la calidad de vida de estos pacientes.

Conclusiones: El ejercicio físico en la sesión de hemodiálisis tiene beneficios para la salud de los pacientes. El ejercicio físico de baja intensidad, así como la elec-

\section{Correspondencia:}

Carmen $\mathrm{M}^{\mathrm{a}}$ Jiménez Prieto

E-mail: carmen_98@hotmail.com troestimulación neuromuscular en las sesiones de diálisis, mejora objetivamente la fuerza muscular, aumenta la capacidad física, mejora la sintomatología depresiva con un mejor manejo del estrés y la ansiedad. Además, perece que puede mejorar el proceso inflamatorio, parámetros nutricionales y disminuir la presión arterial, así como mejorar la calidad de vida. La electroestimulación neuromuscular y el ejercicio físico de baja intensidad, son los ejercicios más utilizados.

PALABRAS CLAVE: hemodiálisis; electroestimulación neuromuscular; ejercicio físico.

\section{Benefits of physical exercise during the haemodialysis session. A systematic review}

\begin{abstract}
Objective: The main objective of this review was to know the main benefits of physical exercise during the dialysis session, in patients in periodic hemodialysis program.
\end{abstract}

Methodology: A systematic review was conducted through the Pubmed, Science Direct and Google Scholar databases. Scientific articles in English and Spanish were included, and articles dealing with physical exercise in the hemodialysis session were analyzed. 
Articles that did not present results and in which physical exercise was at home were excluded.

Results: 18 articles published between 2015 and 2019 were included. All articles were of experimental design. A significant improvement in muscle strength was found, as well as in physical ability and depressive symptomatology. To a lesser extent, a decrease in blood pressure, decrease in the inflammatory process, improvement in nutritional values and improvement in the quality of life of these patients were noticed.

Conclusions: Physical exercise in the hemodialysis session has benefits for the patient's health. Low intensity physical exercise, as well as neuromuscular electro-stimulation in dialysis sessions, objectively improves muscle strength, increases physical capacity, improves depressive symptoms with better management of stress and anxiety. In addition, it seems that it can improve the inflammatory process, nutritional parameters and decrease blood pressure, as well as improving the quality of life. Neuromuscular electro-stimulation and low intensity physical exercise are the most commonly performed exercises.

KEY WORDS: exercise; hemodialysis; neuromuscular electro-stimulation; physical exercise.

\section{Introducción}

La prevalencia de la enfermedad renal crónica en España ha crecido un $20 \%$ en la última década y aumenta de forma progresiva con la edad ${ }^{1}$, constituyendo un importante problema de salud pública. En su último estadio o enfermedad Renal Crónica Avanzada (ERCA) desemboca irreversiblemente en la necesidad de Terapia Renal Sustitutiva (TRS), mediante diálisis 0 trasplante renal. La ERCA presenta en la actualidad una incidencia y prevalencia crecientes, afectando a un porcentaje significativo de la población mundial, debido fundamentalmente, a que sus causas principales residen en trastornos de alta prevalencia como el envejecimiento, la hipertensión arterial, la diabetes y la enfermedad vascular ${ }^{2,3}$. Además del descenso del filtrado glomerular, la ERCA provoca un estado inflamatorio que condiciona cambios fisiológicos con repercusión a nivel de otros órganos ${ }^{4}$.

Esta evolución irreversible de la ERCA condiciona el estadio final de la misma que conduce inexorablemen- te a la necesidad de una TRS. Según datos del Registro Español de Enfermos Renales del año 2018, el 51,5\% de los pacientes con TRS en nuestro país están con un trasplante renal funcionante, mientras que el $42,8 \%$ están en tratamiento con hemodiálisis (HD) en un centro; el $0,15 \%$ en HD domiciliaria, y el 3,3\% en diálisis peritoneal. Además, la prevalencia en pacientes mayores de 64 años es del $25 \%$ y en mayores de 75 años, del $22 \%$; por lo que cada vez hay pacientes más ancianos en $H^{5}$.

Por consiguiente, el perfil actual de la población de pacientes en HD ha cambiado con respecto a hace dos décadas. Con el envejecimiento de la población y las características propias de las TRS, las unidades de diálisis se enfrentan, cada día más, al reto de atender pacientes con un mayor grado de dependencia funcional ${ }^{6}$. A pesar de los avances en el tratamiento de HD, éste no asegura un nivel óptimo de calidad de vida del paciente, por lo que los pacientes en HD presentan una disminución significativa de la calidad de vida relacionada con la salud respecto a la población sana de referencia para la misma edad o a pacientes portadores de un injerto renal funcionante ${ }^{7-9}$.

Derivados de la propia enfermedad e inherentes al procedimiento dialítico, los pacientes en HD presentan una serie de alteraciones asociadas a su enfermedad, con afectación del estado general y diferentes manifestaciones clínicas, siendo especialmente relevantes las alteraciones en el músculo esquelético, con pérdida importante de masa muscular ${ }^{10}$.

Precisamente, las alteraciones musculares son el segundo grupo de alteraciones más importantes presentes en los pacientes en HD, después de las cardiovasculares $^{11}$. Los pacientes con ERCA en tratamiento con HD periódicas que permanecen mucho tiempo en el mismo, sufren alteraciones cuantitativas en su musculatura con una disminución de su masa muscular, con una marcada disminución en la capacidad para realizar trabajo físico, al igual que la tolerancia al ejercicio, en comparación con los sujetos sanos de su misma edad ${ }^{12}$.

Al objeto de paliar este déficit, desde principios de la década de los 80, algunos grupos comenzaron a implantar programas de ejercicio físico de baja intensidad durante la HD, aunque a día de hoy, estos programas de ejercicios durante la sesión de diálisis no se han generalizado ${ }^{13,14}$. No obstante, diferentes estudios han comunicado beneficios de la realización de ejercicio físico durante la HD, tanto a nivel fisiológico, funcional 
o psicológico ${ }^{15}$; aunque en nuestro país no existen muchos grupos que tengan instaurados estos programas, y por tanto hay escasez de investigaciones que analicen los beneficios del ejercicio físico durante la sesión de $H D^{11}$.

Por todo esto, nos parece relevante el estudio de los beneficios del ejercicio físico de baja intensidad en los pacientes con ERCA, durante las sesiones de HD. Por tanto, el objetivo principal de esta revisión fue conocer los principales beneficios del ejercicio físico durante la sesión de diálisis, en los pacientes en programa de HD periódicas, con los siguientes objetivos secundarios:

1.- Identificar las principales variables clínicas que se modifican con el ejercicio físico durante la sesión de HD.

2.- Determinar el tipo de ejercicio utilizado por los pacientes durante las sesiones de HD.

\section{Metodología}

\section{Diseño del estudio}

Se ha realizado una revisión sistemática de la evidencia publicada, generando una síntesis narrativa de los artículos seleccionados. El procedimiento seguido ha sido: búsqueda bibliográfica, selección de artículos, evaluación de calidad, extracción e interpretación de los resultados. La escritura de la revisión sistemática se ha llevado a cabo siguiendo la declaración PRISMA (Preferred Reporting Items for Systematic Reviews and Meta-Analyses) ${ }^{16}$.

\section{Método de búsqueda}

Se ha realizado una búsqueda electrónica en las siguientes bases de datos: Science Direct, Pubmed y Google Académico. Los términos seleccionados para la búsqueda han sido: Ejercicio ("Exercise"), Hemodiálisis ("Hemodialysis"), Ejercicio físico ("Physical exercise"), Electroestimulación Neuromuscular ("Neuromuscular Electroestimulation"). Las palabras clave empleadas fueron las mismas en las diferentes bases.

La búsqueda se realizó entre los meses de diciembre de 2019 a enero de 2020. En la estrategia de búsqueda se combinaron las cuatro palabras-términos clave con el operador booleano AND. La estrategia de búsqueda fue: "Exercise" AND "Hemodialysis", "Physical exercise" AND "Hemodialysis", "Neuromuscular Electroestimulation" AND "Hemodialysis".

\section{Criterios de inclusión/exclusión}

- Criterios de inclusión: estudios observacionales, estudios experimentales o cuasi-experimentales publicados desde el 1 de enero de 2015 hasta la fecha de inicio de esta revisión (diciembre de 2019), sobre ejercicio físico durante la sesión de $H D$, tanto en español como en inglés.

- Criterios de exclusión: estudios en los que el ejercicio se hubiera realizado en el domicilio de los pacientes y estudios en pacientes menores de 18 años.

\section{Resultados de la búsqueda}

Los detalles del proceso de búsqueda se encuentran recogidos en el diagrama de flujo de la Figura 1.

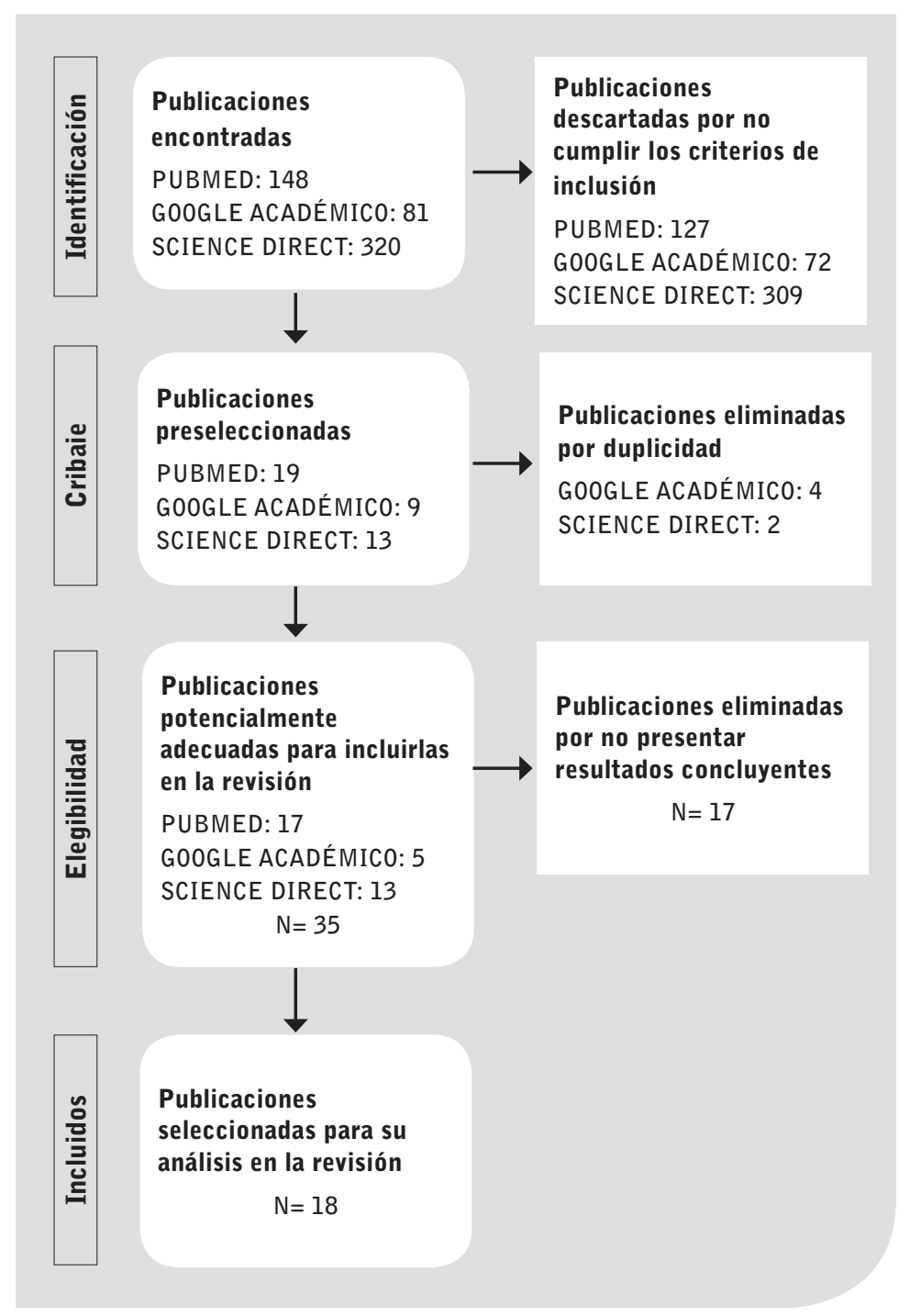

Figura 1. Análisis descriptivo del diagnóstico de infección por COVID-19 en los pacientes en hemodiálisis. 
Se realizó primeramente un cribado mediante la lectura de los títulos y resúmenes de 549 artículos para descartar aquellos que no cumplían con los criterios de inclusión/exclusión. A continuación, se accedió al texto completo de 41 artículos. De ellos, se descartaron 6 por duplicidad y 17 fueron excluidos por no presentar resultados concluyentes. Por último, se seleccionaron 18 artículos para la realización de esta revisión.

\section{Análisis de la calidad de los estudios}

Para evaluar la calidad metodológica de los artículos se utilizó la lista de verificación CASPe ${ }^{17}$ para los estudios experimentales.

\section{Extracción de datos}

Los datos extraídos de cada estudio fueron: fuerza muscular, capacidad física, calidad de vida, presión arterial, inflamación y estrés oxidativo, estado de ánimo y ansiedad-depresión, parámetros bioquímicos y tipo de ejercicio realizado.

\section{Síntesis}

Dieciocho artículos cumplieron los criterios de inclusión. Se empleó un estilo narrativo debido a la heterogeneidad de los estudios y a sus diferencias en cuanto al tipo de factores determinantes de los beneficios del ejercicio físico durante la sesión de diálisis.

\section{Resultados}

Todos los artículos seleccionados fueron de diseño experimental o cuasi-experimental. Así tres estudios fueron cuasi-experimentales, seis de diseño experimental y diez estudios experimentales controlados y randomizados. En la Tabla 1 se muestran las principales características de los artículos seleccionados.

\section{Fuerza muscular}

Diferentes estudios encuentran una mejoría significativa en la fuerza extensión máxima del cuádriceps (FEMQ), aunque no hay cambios significativos en la valoración de la fuerza muscular (fuerza de prensión manual) medida con un dinamómetro homologado (hand-grip) en el brazo dominante $18,20,24,27$. Por el contrario, en otro estudio, se llegó a la conclusión de que después del programa de ejercicio físico intradialítico, además de una mejoría significativa en la FEMQ, se incrementó la fuerza muscular estimada mediante el hand-grip ${ }^{29}$. En el estudio de Junqué Jiménez $A$. et al, no se evidenciaron cambios en la FEMQ al finalizar el estudio, pero sí en la fuerza muscular estimada mediante el hand-grip ${ }^{33}$.

\section{Capacidad física}

Respecto a la capacidad física, medida con el test de la distancia recorrida caminando durante 6 minutos (6MWT), bastantes estudios observan un incremento significativo en la distancia recorrida al finalizar el programa de ejercicio físico ${ }^{18,20,26,27,29,33-35}$.

\section{Calidad de vida}

Algunos artículos destacan una mejoría de la calidad de vida en los pacientes, desde la primera a la última semana, después de un programa de varias semanas de ejercicio intradialítico ${ }^{27,33}$. En cambio, en otro estudio, no se observó ningún cambio relevante en la calidad de vida de estos pacientes después del programa de ejercicio físico adaptado en la sesión de hemodiálisis $^{29}$.

\section{Presión arterial}

Con respecto a la presión arterial, en un estudio encontraron una disminución de la presión arterial diastólica después de semanas en el programa de ejercicio intradialítico ${ }^{19}$; y en otro, se observa una disminución en los valores de presión arterial sistólica después del programa de ejercicio ${ }^{30}$.

\section{Inflamación y estrés oxidativo}

Algunos estudios han documentado que los niveles de inflamación en estos pacientes se redujeron debido al ejercicio intradialítico, después de meses de duración de un programa adaptado ${ }^{21,22}$, en cambio, en otros no se ha evidenciado ninguna disminución del estrés oxidativo ni de la inflamación, disminuyendo incluso la enzima superóxido dismutasa, después del ejercicio físico 23,32 .

\section{Estado de ánimo y ansiedad-depresión}

En algunos de los artículos revisados, se ha llegado a la conclusión de que practicar ejercicio físico intradialítico adaptado y de baja intensidad, ayuda a disminuir la sintomatología depresiva, así como a manejar el estrés y la ansiedad que acarrea la HD crónica junto con la enfermedad renal; influyendo todo esto en una mejora del estado anímico y en el fomento de las relaciones interpersonales $28,29,32,35$.

\section{Parámetros bioquímicos (nivel de urea, estado nutricional)}

Respecto a algunos parámetros bioquímicos, no se han observado cambios en los niveles de urea en sangre en los pacientes, después de un programa de ejercicio intradialítico ${ }^{25,30}$. Otros estudios destacan, una mejoría en los valores nutricionales de estos pacientes ${ }^{21,35}$. 
Tabla 1. Principales características de los artículos seleccionados.

\begin{tabular}{|c|c|c|c|c|c|}
\hline $\begin{array}{l}\text { Autor; año; } \\
\text { país }\end{array}$ & $\begin{array}{l}\text { Tipo de } \\
\text { estudio }\end{array}$ & Muestra & Tipo de ejercicio realizado & Resultados & $\begin{array}{l}\text { Criterios de } \\
\text { calidad }\end{array}$ \\
\hline $\begin{array}{l}\text { Esteve } V \text { et } \\
\text { al. }{ }^{18} ; 2017 \\
\text { España. }\end{array}$ & $\begin{array}{l}\text { Estudio } \\
\text { experimental } \\
\text { unicéntrico. }\end{array}$ & 20 pacientes. & $\begin{array}{l}\text { Electroestimulación } \\
\text { neuromuscular en la sesión de } \\
\text { HD. }\end{array}$ & $\begin{array}{l}\text { El programa mejoró la fuerza muscular, } \\
\text { la capacidad funcional y la composición } \\
\text { muscular del cuádriceps en los pacientes } \\
\text { en HD. }\end{array}$ & $\begin{array}{l}\text { CASPe } \\
(9 / 11)\end{array}$ \\
\hline $\begin{array}{l}\text { Cigarroa I et } \\
\text { al. }{ }^{19 ;} \text { 2016; } \\
\text { Chile. }\end{array}$ & $\begin{array}{l}\text { Estudio } \\
\text { longitudinal, } \\
\text { pre-experimental } \\
\text { sin grupo control, } \\
\text { con un muestreo } \\
\text { no probabilístico. }\end{array}$ & 13 pacientes. & $\begin{array}{l}\text { Ejercicio fuerza-resistencia } \\
\text { muscular en la sesión de HD. }\end{array}$ & $\begin{array}{l}\text { Este programa mejoró la fuerza isométrica } \\
\text { máxima en la extensión de ambas rodillas, } \\
\text { la distancia recorrida en un tiempo } \\
\text { determinado, y se observó una disminución } \\
\text { de la PA diastólica }\end{array}$ & $\begin{array}{l}\text { CASPe } \\
(10 / 11)\end{array}$ \\
\hline $\begin{array}{l}\text { Rubio S et } \\
\text { al. }^{20} ; 2015 \\
\text { España. }\end{array}$ & $\begin{array}{l}\text { Estudio } \\
\text { experimental } \\
\text { unicéntrico, } \\
\text { prospectivo. }\end{array}$ & 13 pacientes. & $\begin{array}{l}\text { Electroestimulación } \\
\text { neuromuscular en la sesión de } \\
\text { HD. }\end{array}$ & $\begin{array}{l}\text { Los resultados del programa fueron: una } \\
\text { mejoría en la fuerza muscular, la capacidad } \\
\text { funcional y la composición muscular del } \\
\text { cuádriceps. }\end{array}$ & $\begin{array}{l}\text { CASPe } \\
(9 / 11)\end{array}$ \\
\hline $\begin{array}{l}\text { Liao M et al. }{ }^{21} \text {; } \\
\text { 2016; Taiwán. }\end{array}$ & $\begin{array}{l}\text { Estudio } \\
\text { experimental } \\
\text { prospectivo } \\
\text { randomizado. }\end{array}$ & 40 pacientes. & $\begin{array}{l}\text { Ejercicio aeróbico de bicicleta } \\
\text { estática en la sesión de HD. }\end{array}$ & $\begin{array}{l}\text { El programa de ejercicio físico alivió la } \\
\text { inflamación y mejoró la nutrición en el } \\
\text { grupo experimental, la densidad mineral del } \\
\text { hueso y la tolerancia al ejercicio. }\end{array}$ & $\begin{array}{l}\text { CASPe } \\
(8 / 11)\end{array}$ \\
\hline $\begin{array}{l}\text { Martin } \mathrm{N} \text { et } \\
\text { al. }{ }^{22} ; 2018 ; \\
\text { Reino Unido. }\end{array}$ & $\begin{array}{l}\text { Estudio } \\
\text { experimental } \\
\text { cruzado } \\
\text { aleatorizado. }\end{array}$ & 11 pacientes. & $\begin{array}{l}\text { Ejercicio aeróbico de bicicleta } \\
\text { estática en la sesión de HD. }\end{array}$ & $\begin{array}{l}\text { En este programa se observó el efecto } \\
\text { antiinflamatorio del ejercicio aeróbico en } \\
\text { bicicleta estática. }\end{array}$ & $\begin{array}{l}\text { CASPe } \\
(8 / 11)\end{array}$ \\
\hline $\begin{array}{l}\text { Bohm J et } \\
\text { al. }{ }^{23} ; 2017 \\
\text { Brasil. }\end{array}$ & $\begin{array}{l}\text { Estudio } \\
\text { experimental } \\
\text { aleatorizado. }\end{array}$ & 30 pacientes. & $\begin{array}{l}\text { Ejercicio aeróbico en bicicleta } \\
\text { estática en la sesión de HD. }\end{array}$ & $\begin{array}{l}\text { Los resultados encontrados fueron: un } \\
\text { aumento de la saturación y la presión } \\
\text { de oxígeno parcial en las personas en } \\
\text { HD, aunque se observó que la capacidad } \\
\text { antioxidante total decreció. }\end{array}$ & $\begin{array}{l}\text { CASPe } \\
(9 / 11)\end{array}$ \\
\hline $\begin{array}{l}\text { Anding } \mathrm{K} \text { et } \\
\text { al. }{ }^{24} ; 2015 ; \\
\text { Alemania. }\end{array}$ & $\begin{array}{l}\text { Ensayo } \\
\text { clínico cuasi } \\
\text { experimental. }\end{array}$ & 46 pacientes. & $\begin{array}{l}\text { Ejercicio de resistencia en la } \\
\text { sesión de HD. }\end{array}$ & $\begin{array}{l}\text { Con este programa se mejoraron los } \\
\text { parámetros de fuerza en el grupo de alta } \\
\text { adherencia, mientras que la fuerza mejoró } \\
\text { considerablemente menos en pacientes } \\
\text { con moderada y baja adherencia al } \\
\text { entrenamiento. }\end{array}$ & $\begin{array}{l}\text { CASPe } \\
(10 / 11)\end{array}$ \\
\hline $\begin{array}{l}\text { Brown PDS } \\
\text { et al. } .^{25} ; 2018 ; \\
\text { Canadá. }\end{array}$ & $\begin{array}{l}\text { Estudio } \\
\text { experimental } \\
\text { clínico cruzado. }\end{array}$ & 22 pacientes. & $\begin{array}{l}\text { Ejercicio de moderada } \\
\text { intensidad en la sesión de HD. }\end{array}$ & $\begin{array}{l}\text { Los resultados de este programa afirmaron } \\
\text { que un mayor ejercicio intradialítico no } \\
\text { induce a un mayor nivel de urea en los } \\
\text { pacientes en HD. }\end{array}$ & $\begin{array}{l}\text { CASPe } \\
(9 / 11)\end{array}$ \\
\hline $\begin{array}{l}\text { Medeiros B } \\
\text { et al.26; 2016; } \\
\text { Brasil. }\end{array}$ & $\begin{array}{l}\text { Estudio } \\
\text { experimental } \\
\text { prospectivo. }\end{array}$ & 14 pacientes. & $\begin{array}{l}\text { Rehabilitación cardiopulmonar } \\
\text { con ejercicio de bicicleta. }\end{array}$ & $\begin{array}{l}\text { Este programa demostró un incremento } \\
\text { progresivo de la distancia recorrida en un } \\
\text { tiempo determinado y una disminución en } \\
\text { el esfuerzo percibido en las personas en HD. }\end{array}$ & $\begin{array}{l}\text { CASPe } \\
(9 / 11)\end{array}$ \\
\hline $\begin{array}{l}\text { De Castro A } \\
\text { et al. }{ }^{27} ; 2018 \text {; } \\
\text { Brasil. }\end{array}$ & $\begin{array}{l}\text { Estudio } \\
\text { experimental } \\
\text { prospectivo } \\
\text { controlado. }\end{array}$ & 43 pacientes. & $\begin{array}{l}\text { Ejercicio de resistencia de } \\
\text { los principales grupos de } \\
\text { músculos (dorsales, pectorales, } \\
\text { cuádriceps y músculos de la } \\
\text { pantorrilla). }\end{array}$ & $\begin{array}{l}\text { Este programa demostró una mejoría } \\
\text { notable en la fuerza muscular, al igual que } \\
\text { en la capacidad física. La calidad de vida } \\
\text { mostró también una importante mejoría. }\end{array}$ & $\begin{array}{l}\text { CASPe } \\
(9 / 11)\end{array}$ \\
\hline $\begin{array}{l}\text { Bennett P et } \\
\text { al. }{ }^{28} ; 2015 \text {; } \\
\text { Australia. }\end{array}$ & $\begin{array}{l}\text { Estudio } \\
\text { experimental. }\end{array}$ & 17 pacientes. & $\begin{array}{l}\text { Realización de yoga en } \\
\text { pacientes en HD. }\end{array}$ & $\begin{array}{l}\text { El yoga demostró ser una forma barata } \\
\text { y accesible de actividad física de baja } \\
\text { intensidad, con potencial para mejorar el } \\
\text { estado de ánimo y disminuir la ansiedad. }\end{array}$ & $\begin{array}{l}\text { CASPe } \\
(9 / 11)\end{array}$ \\
\hline
\end{tabular}


Tabla 1. Principales características de los artículos seleccionados.

\begin{tabular}{|c|c|c|c|c|c|}
\hline $\begin{array}{l}\text { Autor, año, } \\
\text { país }\end{array}$ & $\begin{array}{l}\text { Tipo de } \\
\text { estudio }\end{array}$ & Muestra & Tipo de ejercicio realizado & Resultados & $\begin{array}{l}\text { Criterios de } \\
\text { calidad }\end{array}$ \\
\hline $\begin{array}{l}\text { Esteve } V \text { et } \\
\text { al.29; 2015; } \\
\text { España. }\end{array}$ & $\begin{array}{l}\text { Estudio cuasi } \\
\text { experimental } \\
\text { unicéntrico. }\end{array}$ & 22 pacientes. & $\begin{array}{l}\text { Programa adaptado de ejercicio } \\
\text { físico de baja intensidad } \\
\text { mediante pelotas medicinales, } \\
\text { pesas, bandas elásticas y } \\
\text { cicloergómetros en las primeras } \\
2 \mathrm{~h} \text { de diálisis. }\end{array}$ & $\begin{array}{l}\text { Con el programa de ejercicio físico, se } \\
\text { mejoró la fuerza muscular, la capacidad } \\
\text { funcional, así como la sintomatología } \\
\text { depresiva y la calidad de vida. }\end{array}$ & $\begin{array}{l}\text { CASPe } \\
(10 / 11)\end{array}$ \\
\hline $\begin{array}{l}\text { Spósito R et } \\
\text { al. }{ }^{30} ; 2016 ; \\
\text { Brasil. }\end{array}$ & $\begin{array}{l}\text { Estudio } \\
\text { experimental } \\
\text { prospectivo } \\
\text { aleatorizado. }\end{array}$ & 40 pacientes. & $\begin{array}{l}\text { Electroestimulación } \\
\text { neuromuscular en la sesión de } \\
\text { HD. }\end{array}$ & $\begin{array}{l}\text { Los pacientes aumentaron la presión } \\
\text { máxima inspiratoria y la espiratoria. } \\
\text { También aumentó la distancia recorrida } \\
\text { caminando en } 6 \text { minutos y la disminución de } \\
\text { su presión sistólica. }\end{array}$ & $\begin{array}{l}\text { CASPe } \\
(8 / 11)\end{array}$ \\
\hline $\begin{array}{l}\text { McAdams-De- } \\
\text { Marco M et } \\
\text { al. }^{31} ; 2017 \\
\text { EEUU. }\end{array}$ & $\begin{array}{l}\text { Ensayo } \\
\text { experimental } \\
\text { aleatorizado y } \\
\text { controlado. }\end{array}$ & 20 pacientes. & $\begin{array}{l}\text { Para la función cognitiva } \\
\text { realizaban } 10 \text { juegos diferentes } \\
\text { en cada sesión de HD, con } \\
\text { pedales estáticos en cada sesión } \\
\text { de HD. }\end{array}$ & $\begin{array}{l}\text { El declive cognitivo en la velocidad } \\
\text { psicomotora y en la función de ejecución } \\
\text { observado en los resultados del programa, } \\
\text { posiblemente pueda prevenirse por un } \\
\text { entrenamiento cognitivo y un entrenamiento } \\
\text { de ejercicio. }\end{array}$ & $\begin{array}{l}\text { CASPe } \\
(10 / 11)\end{array}$ \\
\hline $\begin{array}{l}\text { Esgalhado M } \\
\text { et al. } .^{32} ; 2015 ; \\
\text { Brasil. }\end{array}$ & $\begin{array}{l}\text { Estudio } \\
\text { experimental } \\
\text { prospectivo. }\end{array}$ & 16 pacientes. & $\begin{array}{l}\text { Ejercicio de fuerza y resistencia } \\
\text { con bandas elásticas y puños } \\
\text { de tobillo en los miembros } \\
\text { inferiores. }\end{array}$ & $\begin{array}{l}\text { El programa mostró una disminución } \\
\text { importante del superóxido dismutasa, lo que } \\
\text { podría incrementar el estrés oxidativo en } \\
\text { los pacientes en HD. }\end{array}$ & $\begin{array}{l}\text { CASPe } \\
(9 / 11)\end{array}$ \\
\hline $\begin{array}{l}\text { Junqué A et } \\
\text { al.33; 2015; } \\
\text { España. }\end{array}$ & $\begin{array}{l}\text { Estudio } \\
\text { experimental } \\
\text { prospectivo. }\end{array}$ & 11 pacientes. & $\begin{array}{l}\text { Ejercicio físico adaptado } \\
\text { mediante pesas, pelotas } \\
\text { medicinales, bandas elásticas y } \\
\text { cicloergómetros. }\end{array}$ & $\begin{array}{l}\text { El programa mejoró la fuerza muscular, la } \\
\text { capacidad funcional y la calidad de vida de } \\
\text { pacientes ancianos en HD. }\end{array}$ & $\begin{array}{l}\text { CASPe } \\
(9 / 11)\end{array}$ \\
\hline $\begin{array}{l}\text { Abdelaal } \\
\text { A.A.M et al. }{ }^{34} \text {; } \\
\text { 2019; Egipto. }\end{array}$ & $\begin{array}{l}\text { Estudio } \\
\text { experimental } \\
\text { aleatorizado. }\end{array}$ & 66 pacientes. & $\begin{array}{l}\text { Programa de ejercicio } \\
\text { aeróbico y otro de ejercicio de } \\
\text { resistencia. }\end{array}$ & $\begin{array}{l}\text { Este programa mejoró notablemente el } \\
\text { equilibrio funcional y la capacidad física. }\end{array}$ & $\begin{array}{l}\text { CASPe } \\
(9 / 11)\end{array}$ \\
\hline $\begin{array}{l}\text { Dashtidehkor- } \\
\text { di A et al. }{ }^{35} \text {; } \\
\text { 2019; Iran. }\end{array}$ & $\begin{array}{l}\text { Estudio } \\
\text { experimental } \\
\text { aleatorizado. }\end{array}$ & 60 pacientes. & $\begin{array}{l}\text { Ejercicio físico adaptado } \\
\text { utilizando una bicicleta estática. }\end{array}$ & $\begin{array}{l}\text { El ejercicio físico fue efectivo para la } \\
\text { mejora de algunas áreas como la física, el } \\
\text { estado de nutrición, el manejo del estrés, y } \\
\text { las relaciones interpersonales. }\end{array}$ & $\begin{array}{l}\text { CASPe } \\
(10 / 11)\end{array}$ \\
\hline
\end{tabular}

\section{Tipo de ejercicio}

En cuanto al tipo de ejercicio incluido en los diferentes programas de ejercicio durante la sesión de diálisis, se encontraron:

- Electroestimulación neuromuscular ${ }^{18,20,30}$ : en los cuádriceps, mediante la colocación de electrodos en el punto motor de los vientres musculares del cuádriceps. El paciente se encuentra en decúbito supino, con extensión completa de los miembros inferiores y mínima flexión de ambas rodillas mediante una almohada blanda colocada en la región poplítea. El programa de electroestimulación incluía: un programa de tonificación en la primera semana, una semana de resistencia aeróbica, 2 semanas de rehabilitación-hipertrofia, 3 semanas de potenciación muscular, y 3 semanas de fuerza-resistencia. Se realiza durante las primeras 2 horas de la sesión de hemodiálisis ${ }^{18,20}$. En cambio, en la electroestimulación neuromuscular descrita por Spósito R. y colaboradores ${ }^{30}$, el tiempo de electroestimulación descrita en su estudio duraba solamente 30 minutos del tiempo ocupado en la hemodiálisis diaria. Aun así, los resultados en ambos son similares: mejora de la fuerza muscular, la capacidad funcional y la fuerza extensión máxima del cuádriceps.

- Ejercicio físico aeróbico de baja intensidad 19,21-29,31-35, entre los que podemos distinguir tres tipos de ejercicio: el realizado aeróbicamente en una bicicleta estática o en pedales estáticos $21-23,26,31,35$, el ejercicio de 
fuerza-resistencia en el que los pacientes realizaban ejercicio físico con su propio cuerpo, trabajando diferentes grupos musculares, tanto miembros superiores como miembros inferiores ${ }^{19,24,25,27,28,34}$, y en el que los pacientes realizaban ejercicio físico de baja intensidad con pesas, pelotas medicinales, bandas elásticas y cicloergómetros ${ }^{29,32,33}$; siendo el mejor tolerado por los pacientes el ejercicio en bicicleta estática de baja intensidad o con pedales estáticos, pues los pacientes se encuentran sentados mientras realizan ejercicio físico, y su cansancio tarda más en llegar y es menor que en otros tipos de ejercicio.

\section{Discusión}

En gran parte de los artículos utilizados para esta revisión, los autores destacan que uno de los beneficios más significativos del ejercicio físico en HD es la mejora de la fuerza muscular. Estos resultados, concuerdan con gran parte de la bibliografía existente al respecto, en la que diferentes autores encuentran similares resultados ${ }^{40-49}$. Otros autores han destacado la mejoría en la FEMQ y la fuerza estimada mediante el hand-grip ${ }^{41-43,45-48}$, aunque en nuestra revisión solo encontramos un artículo que analizara estos parámetros por separado ${ }^{29}$. Sin embargo, un autor encontró una mejoría en la fuerza muscular de los cuádriceps, y no en la fuerza mediante el handgrip $^{15}$.

También se han encontrado beneficios constatados en algunos de los resultados de esta revisión, en la mejora de la capacidad física, coincidiendo estos resultados con los otros autores ${ }^{36-43,45-50}$, los cuales afirman una mejora significativa de la capacidad física después del ejercicio de baja intensidad programado en HD.

Por otra parte, también es destacable el beneficio del ejercicio físico intradialítico en el estado de ánimo y ansiedad-depresión; poniéndose de manifiesto, una mejora significativa del estado de depresión por parte de los pacientes que realizan ejercicio de baja intensidad programado en la sesión de hemodiálisis, en la misma línea que han documentado otros autores $37,40,42,45,46,48$.

Algunos autores han encontrado una reducción de la presión arterial ambulatoria de estos pacientes, sin incidencia en la presión arterial durante la sesión, como beneficio del ejercicio intradialítico, en la línea de lo publicado por otros estudios ${ }^{36,38,50}$, aunque no son muchos estudios y sin resultados coincidentes. En el artículo de Henrique DMN et $\mathrm{al}^{36}$ y Alonso MR et $\left.a\right|^{50}$, se observaba una disminución de la presión arterial sistólica y diastólica, mientras que en otro artículo ${ }^{38}$, la disminución se observaba solamente en la presión sistólica.

Otro de los beneficios destacado por varios autores, es la mejoría de la calidad de vida del paciente en HD, como consecuencia de este ejercicio programado intradialítico; algo que ya habían destacado diferentes autores ${ }^{42,44,46,49}$; utilizando la mayoría de ellos, para la evaluación de la calidad de vida, el instrumento validado EuroQol-5D.

Otro parámetro que ha mejorado con el ejercicio físico ha sido el estado inflamatorio, que suele acompañar a estos pacientes. En efecto, en algunos estudios se ha observado que los niveles de marcadores de la inflamación se redujeron debido al ejercicio intradialítico, después de meses de duración de un programa adaptado ${ }^{21,22}$; aunque estos resultados no son muy concluyentes ni hemos encontrado bibliografía para poder compararlos, igual que ocurre con los estudios que destacan una mejoría en los valores nutricionales de estos pacientes, como consecuencia del ejercicio durante la sesión de HD.

No se han encontrado modificaciones destacables en cuanto a otros parámetros bioquímicos en esta revisión, al contrario que en el estudio de Alonso MR et al ${ }^{50}$, en el que se encuentra una diminución de los niveles de urea en sangre después del ejercicio programado de baja intensidad en la sesión de HD.

Con respecto al tipo de ejercicio que se ha utilizado en los artículos encontrados, tenemos que hablar de dos tipos: electroestimulación neuromuscular y ejercicio físico de baja intensidad. Con respecto a ellos, no se ha encontrado estudios que afirmen qué tipo de ejercicio es mejor que el otro, sino que son utilizados uno u otro indistintamente en los estudios realizados. Aun así, en los artículos encontrados, hay una gran variación en el número de estudios que han utilizado ejercicio físico de baja intensidad y la electroestimulación neuromuscular. En efecto, el número de artículos que utilizan un programa de ejercicio físico de baja intensidad es mucho mayor que el número de artículos que han utilizado la electroestimulación neuromuscular, aunque en ningún momento se habla de cuál de ellos es mejor que el otro.

\section{Limitaciones del estudio}

Las principales limitaciones de este estudio han sido la falta de publicaciones referidas al ejercicio físico de baja intensidad en la sesión de HD, pues muchos de los 
estudios encontrados, hacían referencia a programas de ejercicio físico domiciliario en pacientes que llevaban en HD varios años, y no hablaban concretamente del ejercicio realizado dentro de sus sesiones de HD. Por otro lado, el número de estudios encontrados en los que el tipo de ejercicio utilizado es el ejercicio físico de baja intensidad, ha sido muy superior a los encontrados en los que el tipo de ejercicio utilizado es la electroestimulación neuromuscular.

\section{Consideraciones prácticas}

A pesar de que la bibliografía encontrada en los últimos 5 años no es muy amplia, de ella se desprende que el ejercicio físico de baja intensidad y la electroestimulación neuromuscular durante la sesión de diálisis comportan varios efectos beneficiosos en los pacientes en HD, especialmente los que pueden colaborar a mitigar la debilidad muscular presente en estos pacientes, especialmente en los pacientes añosos. Por ello, puede ser importante instaurar estos programas de ejercicio físico en las sesiones de HD, pues disminuiría su sedentarismo, además de incrementar su masa muscular, su capacidad física, y por ende, su calidad de vida.

A la vista de estos resultados podemos concluir que en general, el ejercicio físico de baja intensidad, así como la electroestimulación neuromuscular durante las sesiones de diálisis, mejoran objetivamente la fuerza muscular, aumenta la capacidad física, mejoran la sintomatología depresiva con un mejor manejo del estrés y la ansiedad, en los pacientes en HD. Además, parece que este ejercicio puede mejorar el proceso inflamatorio, parámetros nutricionales y disminuir la presión arterial, así como mejorar la calidad de vida.

El ejercicio físico más realizado en los diferentes programas revisados son la electroestimulación neuromuscular y el ejercicio físico aeróbico de baja intensidad, entre los que destacan el realizado en bicicleta estática o pedales estáticos, el de fuerza-resistencia en el que los pacientes realizan ejercicio físico con su propio cuerpo trabajando diferentes grupos musculares y el ejercicio físico de baja intensidad con pesas, pelotas medicinales, bandas elásticas y cicloergómetros.

Recibido: 15-07-20

Revisado: 27-07-20

Modificado: 03-08-20

Aceptado: 05-08-20

\section{Bibliografía}

1. Documento Marco sobre Enfermedad Renal Crónica (ERC) dentro de la Estrategia de Abordaje a la Cronicidad en el SNS. Febrero 2015. Subdirección General de Calidad y Cohesión. [Consultado 07 mar 2020]. Disponible en: https://www.mscbs.gob.es/ organizacion/sns/planCalidadSNS/pdf/Enfermedad_ Renal_Cronica_2015.pdf.

2. De Francisco $A$, Sanjuán $F$, Foraster $A$, Fabado $S$, Carretero D, Santamaría C, et al. Estudio epidemiológico de pacientes ancianos con insuficiencia renal crónica en hemodiálisis. Nefrología. 2008;28:48-55.

3. Soriano Cabrera S. Definición y clasificación de los estadios de la enfermedad renal crónica. Prevalencia. Claves para el diagnóstico precoz. Factores de riesgo de enfermedad renal crónica. Nefrología. 2004;24:27-34.

4. Portilla-Franco $M$, Tornero-Molina F, Gil-Gregorio P. La fragilidad en el anciano con enfermedad renal crónica. Nefrología. 2016;36(6):609-15.

5. Registro Español de Enfermos Renales. Informe de Diálisis y Trasplante 2018. [Consultado 07 mar 2020]. Disponible en: https://www.senefro.org/contents/webstructure/SEN_2019_REER_modificada. pdf.

6. Arenas-Jiménez M, Navarro-García M, Serrano-Reina E, Álvarez-Ude F. Dependencia para las actividades instrumentales de la vida diaria en pacientes en hemodiálisis. Nefrología. 2019;39(5):531-8.

7. Albañil-Frías T, Ramírez-Moreno MC, Crespo-Montero R. Análisis de la calidad de vida del paciente en hemodiálisis ambulatoria y su relación con el grado de dependencia. Enferm Nefrol. 2014;17(3):16774.

8. Adell-Lleixà M, Casadó-Marin L, Andújar-Solé J, Solà-Miravete E, Martínez-Segura E, Salvadó-Usach T. Valoración de la calidad de vida relacionada con la salud en la enfermedad renal crónica terminal mediante un cuestionario de resultados percibidos por los pacientes. Enferm Nefrol. 2016;19(4):331-40.

9. Merino-Martínez RM, Morillo-Gallego N, Sánchez-Cabezas AM, Gómez-López V, Crespo-Monte- 
ro R. Relación entre la calidad de vida relacionada con la salud y la ansiedad/depresión en pacientes en hemodiálisis crónica. Enferm Nefrol. 2019;22(3): 274-83.

10. Roshanravan B, Gamboa J, Wilund K. Exercise and CKD: Skeletal muscle dysfunction and practical application of exercise to prevent and treat physical impairments in CKD. Am J Kidney Dis. 2017;69:837-52.

11. Segura-Ortí E. Fisioterapia durante la hemodiálisis: resultados de un programa de fuerza-resistencia. Nefrología. 2008;28(1):67-72.

12. Young-Seong E. Acute intradyalitic exercise and oxidative stress in hemodialysis patients. Kidney Res Clin Pract. 2015;34:1-3.

13. Junqué-Jiménez $A$, Esteve-Simó $V$, Iza-Pinedo $G$, Bernabéu E, Luceño Soler I, Paz López 0, et al. Resultados de un programa de ejercicio físico combinado con electroestimulación neuromuscular en pacientes en hemodiálisis. Enferm Nefrol. 2013; 16(3):161-7.

14. Ortega-Pérez de Villar L, Antol-García S, Lidón-Pérez $\mathrm{M}^{\mathrm{a}} \mathrm{J}$, Amer-Cuenca J.J, Benavent-Caballer V, Segura-Ortí E. Comparación de un programa de ejercicio intradiálisis frente a ejercicio domiciliario sobre capacidad física funcional y nivel de actividad física. Enferm Nefrol. 2016;19(1):45-54.

15. Moreno-Arroyo M, Hidalgo-Blanco M. El ejercicio físico y el paciente renal crónico. Enferm Nefrol. 2012;15(4):296-9.

16. Urrútia G, Bonfill X. Declaración PRISMA: una propuesta para mejorar la publicación de revisiones sistemáticas y metaanálisis. Med Clin. 2010; 135(11):507-11.

17. Cabello JB, por CASPe. Plantilla para ayudarte a entender un Ensayo Clínico. En: CASPe. Guías CASPe de Lectura Crítica de la Literatura Médica. Alicante: CASPe; 2005. Cuaderno I. p.5-8. [Internet]. [Consultado 07 mar 2020]. Disponible en: http://www.redcaspe.org/herramientas/instrumentos.

18. Esteve V, Carneiro J, Moreno F, Fulquet M, Garriga $\mathrm{S}$, Pou $\mathrm{M}$, et al. Efecto de la electroestimulación neuromuscular sobre la fuerza muscular, capacidad funcional y composición corporal en los pacientes en hemodiálisis. Nefrología. 2017;37(1):68-77.

19. Cigarroa I, Barriga R, Michéas C, Zapata-Lamana $R$, Soto C, Manukian T. Efectos de un programa de ejercicio de fuerza-resistencia muscular en la capacidad funcional, fuerza y calidad de vida de adultos con enfermedad renal crónica en hemodiálisis. Rev Med Chile. 2016;144:844-52.

20. Rubio-Páez $S$, Esteve-Simó $V$, Junqué-Jiménez $A$, Tomás-Bernarbéu E, Paz-López 0, Iza-Pinedo 0 et al. Electroestimulación neuromuscular intradiálisis, fuerza muscular, capacidad funcional y composición corporal. Enferm Nefrol. 2015;18(4):273-81.

21. Liao MT, Liu WCh, Lin FH, Huang ChF, Chen SY, $\mathrm{Liu} \mathrm{Ch}$, et al. Intradyalitic aerobic cycling exercise alleviates inflammation and improves endothelial progenitor cell count and bone density in hemodialysis patients. Medicine. 2016;95(27):e4134.

22. Martin N, Smith A, Dungey $M$, Young $H$, Burton $J$, Bishop N. Exercise during hemodialysis does not affect the phenotype or prothrombotic nature of microparticles but alters their proinflammatory function. Physiol Rep. 2018;6(19):1-13.

23. Bohm J, Borba-Monteiro M, Porcher-Andrade $F$, Veríssimo-Veronese F, Saldanha-Thomé F. Acute effects of intradialytic aerobic exercise on solute removal, blood gases and oxidative stress in patients with chronic kidney disease. J Bras Nefrol. 2017;39(2):172-80.

24. Anding K, Bar T, Trojniak-Hennig J, Kuchinke S, Krause R, Rost J.M, et al. A structured exercise programme during haemodialysis for patients with chronic kidney disease: clinical benefit and longterm adherenc. BMJ Open. 2015;5:1-10.

25. Brown PDS, Rowed K, Shearer J, McRae J, Parker K. Impact of intradialytic exercise intensity on urea clearance in hemodialysis patients. Appl. Physiol. Nutr. Metab. 2018;43:101-4.

26. Medeiros-Guio B, Pérez-Gomes C, Brandao da Costa $F$, dos Santos de Oliveira A, Turano-Duarte $M$, Leite-Júnior M. Beneficial effects of intradialytic cardiopulmonary rehabilitation. J Bras Nefrol. 2017;39(3):275-82. 
27. André de Castro AP, Ribeiro-Barbosa $S$, Novais-Mansur $\mathrm{H}$, Andrade-Ezequiel DG, Barros-Costa M, Baumgratz de Paula R. Intradialytic resistance training: an effective and easy-to execute strategy. J Bras. Nefrol. 2018;41(2):215-23.

28. Bennet PN, Parsons T, Ben-Moshe R, Neal M, Weinberg MK, Gilbert K. Intradialytic Laughter Yoga therapy for haemodialysis patients: a pre-post intervention feasibility study. BMC Complementary and Alternative Medicine. 2015;15:176; doi: 10.1186/ s12906-015-0705-5.

29. Esteve-Simó $V$, Junqué-Jiménez $A$, Moreno-Guzmán F, Carneiro-Oliveira J, Fulquet-Nicolas M, Po-Potau $M$. Beneficios del ejercicio físico de baja intensidad durante la sesión de hemodiálisis en el paciente anciano. Nefrología. 2015;35(4):385-94.

30. Spósito-Roxo R, Bertoni-Xavier V, Antonio-Miorin L, Olivares-Magalhaes A, Santos-Sens YA, Santos-Alves VL. Impact of neuromuscular electrical stimulation on functional capacity of patients with chronic kidney disease on hemodialysis. J Bras Nefro. 2016; 38(3):344-50.

31. McAdams MA, Konel J, Warsame F, Ying H, González-Fernández M, Carlson MC. Intradialytic cognitive and exercise training may preserve cognitive function. Kidney International Reports. 2018;3:81-8.

32. Esgalhado M, Barcza M, Ferreira-Medeiros L, Costa C, Barboza JE, Mafra D. Effects of accute intradialytic strenght physical exercise on oxidative stress and inflammatory responses in hemodialysis patients. Kidney Res Clin Pract. 2015;34:35-40.

33. Junqué-Jiménez $A$, Esteve-Simó $V$, Tomás-Bernabéu E, Paz-López 0, Iza-Pinedo G, Luceño-Solé I. Resultado de un programa adaptado de ejercicio físico en pacientes ancianos en hemodiálisis. Enferm Nefrol. 2015;18(1):11-8.

34. Mohamed-Abdelaal AA, Mohamed-Abdulaziz E. Effect of exercise therapy on physical performance and functional balance in patients on maintenance renal hemodialysis: randomized controlled study. Journal of Exercise Rehabilitation. 2019;15(3):472-80.

35. Dashtidehkordi A, Shahgholian N, Attari F. Exercise during hemodialysis and health promoting behaviors: a clinical trial. BMC Nephrology. 2019;20:96.
36. Henrique DMN, Reboredo $M$ de $M$, Chaoubah $A$, Paula RB. Aerobic Exercise Improves Physical Capacity in Patients under Chronic Hemodialysis. Arq Bras Cardiol. 2010;94:823-8.

37. Fernández-Lara MJ, Ibarra-Cornejo JL, Aguas-Alveal EV, González-Tapia CE, Quidequeo-Reffers DG. Revisión: Beneficios del ejercicio físico en pacientes con enfermedad renal crónica en hemodiálisis. Enferm Nefrol. 2018;21(2):167-81.

38. Pu J, Jiang Z, Wu W, Li L, Zhang L, Li Y, Liu Q, Ou S. Efficacy and safety of intradialytic exercise in haemodialysis patients: a systematic review and meta-analysis. BMJ Open. 2019;9:1-12.

39. Capitanini A, Lange S, D Alessandro C, Salotti E, Tavolaro $A$, Baronti ME, et al. Dialysis Exercise Team: The Way to Sustain Exercise Programs in Hemodialysis Patients. Kidney Blood Press Res. 2014; 39:129-33.

40. Yon-Rhee S, Kyung-Song J, Chul-Hong S, Won-Choi J, Jung-Jeon $\mathrm{H}, \mathrm{Ho}$-Shin $\mathrm{D}$, et al. Intradialytic exercise improves physical function and reduces intradialytic hypotension and depression in hemodialysis patient. Korean J Intern Med. 2019;34: 588-98.

41. Junqué-Jiménez $A$, Esteve-Simón $V$, Iza-Pinedo $G$, Tomás Bernabéu E, Luceño-Soler I, Paz-López 0 et al. Resultados de un programa de ejercicio físico combinado con electroestimulación neuromuscular en pacientes en hemodiálisis. Enferm Nefrol. 2013; 16(3):161-7.

42. Tomás-Bernabéu $E$, Junqué-Jiménez $A$, Iza-Pinedo G, Luceño-Solé I, Paz-López 0, Lavado-Santpere M. Beneficios de un programa de entrenamiento físico en la sintomatología depresiva y calidad de vida en los pacientes en hemodiálisis. Enferm Nefrol. 2013; 16(Suppl 1):170-1.

43. Junqué-Jiménez $A$, Tomás-Bernabéu $E$, Iza-Pinedo G, Luceño-Solé I, Paz-López 0, Lavado-Santpere M. Ejercicio físico, parámetros nutricionales y composición corporal en pacientes en hemodiálisis. Enferm Nefrol. 2013;16(Suppl 1):178-9.

44. Contreras-Martos G.M, Delgado-Rodríguez M, Martínez-Villar J, Parra-Mozas I, Borrego-Utiel F, Segura-Torres $\mathrm{P}$. Eficacia de un programa de entrenamiento intradiálisis de fuerza-resistencia en combinación 
con electroestimulación neuromuscular: mejora en la capacidad funcional, fuerza, y calidad de vida. Rev Soc Esp Enferm Nefrol. 2011;14(2):112-9.

45. Moreno-Enríquez $L$, Junqué-Jiménez $A$, Esteve-Simón V, Lavado-Santpere M, Ramírez de Arellano M. Entrenamiento físico en hemodiálisis: efectos sobre la fuerza muscular, capacidad funcional, depresión y calidad de vida. Enferm Nefrol. 2011;15(Suppl 1):111.

46. Junqué-Jiménez $A$, Tomás-Bernaveu $E$, Iza-Pinedo G, Paz-López 0, Luceño-Solé I, Lavado-Sempere M. Resultados de un programa adaptado de ejercicio físico en pacientes ancianos ( $>75$ años) en hemodiálisis. Enferm Nefrol. 2014;17(Suppl 1):71.

47. Segura-Ortí E. Ejercicio en pacientes en hemodiálisis: revisión sistemática de la literatura. Nefrología. 2010;30(2):236-46.

48. Moreno-Enríquez $L$, Junqué-Jiménez $A$, Esteve-Simón V, Lavado-Santpere M, Ramírez de Arellano M, Tomás-Bernabéu E. Mejoría de la sintomatología depresiva mediante un programa de ejercicio físico en pacientes en hemodiálisis. Enferm Nefrol. 2012;15 (Suppl 1):62-3.
49. Junqué-Jiménez $A$, Esteve-Simó $V$, Tomás-Bernaveu E, Paz-López 0, Iza-Pinedo 0, Luceño-Solé I, y cols. Electroestimulación neuromuscular: una nueva opción terapéutica en la mejoría de la condición física de los pacientes en hemodiálisis. Enferm Nefrol. 2014;17(4):269-76.

50. Alonso MR, Midley AD, Crucelegui M S, Patiño 0, Galarza Hangling MM, Pjillipi $R$, et al. Evaluación de un programa de actividad física intradialítica en pacientes con hemodiálisis. Nefrol Lationam. 2017; 14(1):4-11.

Este artículo se distribuye bajo una Licencia Creative Commons Atribución-NoComercial 4.0 Internacional. https://creativecommons.org/licenses/by-nc/4.0/ 